\title{
A prospective trial of GreenLight PVP (HPS120) versus transurethral resection of the prostate in the treatment of lower urinary tract symptoms in Ontario, Canada
}

\author{
J. Paul Whelan BSc, MD, FRCSC,* James M. Bowen, BScPhm, MSc, ${ }_{r}^{+}$Natasha Burke, MSc, ${ }^{\dagger}$ Edward A. Woods \\ MD, FRCSC,; Gary P. Mclssac, BSc, MD, FRCSC;: Robert B. Hopkins, PhD; ${ }_{;}^{+}$Daria J. O’Reilly, PhD; Feng Xie, \\ PhD; ${ }^{+}$Shayan Sehatzadeh, MD, MSc,; Leslie Levin, MD, FRCP(Lond), FRCPC,; Suja P. Mathew, BDS,; Lisa L. \\ Patterson, $B A^{;}{ }^{\dagger}$ Ron Goeree, $M A^{;}{ }^{+}$Jean-Eric Tarride, $\mathrm{PhD}^{+}$
}

*Division of Urology, McMaster University, Hamilton, ON; 'Programs for Assessment of Technology in Health (PATH) Research Institute, St. Joseph's Healthcare Hamilton and Department of Clinical Epidemiology \& Biostatistics, Faculty of Health Sciences, McMaster University, Hamilton, ON; §The Scarborough Hospital, Scarborough, ON; \pm Trillium Health Centre, Mississauga, ON; *Health Quality Ontario, Toronto, ON

Cite as: Can Urol Assoc J 2013;7(9-10):335-41. http://dx.doi.org/10.5489/cuaj.180 Published online October 7, 2013.

\section{Abstract}

Background: Photoselective vaporization of the prostate (PVP) is a bloodless, relatively painless alternative to transurethral resection of the prostate (TURP) for relief of lower urinary tract symptoms (LUTS) in benign prostatic hyperplasia (BPH).

Objective: We compare the effectiveness, safety and cost-effectiveness of Greenlight Laser PVP (HPS-120) and TURP.

Methods: We conducted a prospective, non-randomized trial in 3 Ontario centres from March 2008 to February 2011. Assessments were completed at baseline, 1 and 6 months following surgery at the physicians' offices and at 12 and 24 months by phone. The primary outcome was the change in International Prostate Symptoms Score (IPSS) score at 6 months versus baseline. Secondary outcomes were changes in flow rate, postvoid residual (PVR), prostate-specific antigen (PSA) and sexual health inventory for men (SHIM) scores. Adverse events, health-related quality of life (HRQoL), resource utilization and productivity losses were collected.

Results: Although the IPSS decreased in both arms ( $n=140$ for PVP and $n=24$ for TURP) between baseline and 6 months, the difference in change over time between the groups was not statistically significant ( $p=0.718)$. Other outcomes improved equally from baseline and 6 months (Qmax, SHIM, PSA and HRQoL), with only changes in PVR favouring PVP $(p=0.018)$. There were no statistical differences in serious adverse events. In total, 130 of 140 PVP patients were outpatients, all TURP subjects were inpatients. PVP was less costly than TURP ( $\$ 3891$ vs. $\$ 4863 ; p<0.001$ ) with similar quality-adjusted life years $(0.448$ vs. $0.441 ; p=0.658)$. Conclusion: Greenlight Laser PVP (HPS-120) is a safe and costeffective alternative to TURP for outpatient treatment of LUTS and can be completed as an outpatient with minimal blood loss.

\section{Introduction}

Since its introduction in the 1930s, transurethral resection of the prostate (TURP) has been the gold standard to treat lower urinary tract symptoms (LUTS) secondary to benign prostatic hyperplasia (BPH). However, recent innovations in energy-based interventions have provided alternative treatment options for patients with BPH and may have clinical and economic benefits when compared to TURP. ${ }^{1,2}$ Among these technologies, the potassium-titanyl-phosphate laser, which permits the photoselective vaporization of the prostate (PVP), has the potential to be cost-effective compared to TURP; it appears to provide similar outcomes ${ }^{3-5}$ at a lesser $\cos ^{2,6,7}$ and can be performed in an outpatient setting. Due to the potential benefits associated with this technology and upon review of the evidence, in 2007 the Ontario Health Technology Advisory Committee (OHTAC) ${ }^{8}$ recommended that "a registry study be conducted to establish longer term effectiveness and complication rates for PVP given the likelihood of increasing diffusion of this technology." ${ }^{9}$ The following study was designed to respond to OHTAC's request for further information regarding PVP.

The primary objective of the study was to compare the effectiveness of GreenLight HPS-120 and TURP in the treatment of BPH as measured by the change in International Prostate Symptoms Score (IPSS) ${ }^{10}$ at 6 months following intervention. Secondary objectives were to: (a) determine intra- and postoperative complication rates; (b) compare the 2 treatments at 6 months using other standard clinical outcomes (e.g., urine flow rate, postvoid residual volumes [PVR], quality of life, sexual function); (c) determine 6-month resource utilization and costs of treating patients with PVP and TURP; (d) evaluate the 6-month cost-effectiveness of PVP versus TURP; and (e) evaluate the durability of PVP at 12 and 24 months. 
Whelan et al.

\section{Methods}

\section{Study overview}

A prospective non-randomized trial was conducted at 3 centres across Ontario (St. Joseph's Healthcare Hamilton, Trillium Health Centre and the Scarborough Hospital). The study protocol and consent process were approved by the ethics board of each institution. Patients were identified by their urologist as candidates for TURP and referred to the study. In Phase I of the study, subjects were evaluated at baseline and at months 1 and 6 following surgery. In the second phase of the study, subjects were contacted by telephone at 12 and 24 months to evaluate the long-term durability of the procedures and the subjects' health-related quality of life (HRQoL).

\section{Inclusion and exclusion criteria}

Consenting subjects were screened for inclusion and exclusion criteria. Exclusion criteria are provided in detail in Appendix 1, http://journals.sfu.ca/cuaj/index.php/journal/ article/view/180/1354. Although patients already taking alpha-blockers and/or 5-alpha-reductase inhibitors could be enrolled in the study, these medications were discontinued postoperatively.

The following were the eligibility criteria:

- male and over 40 years old.

- diagnosed with symptomatic/obstructive symptoms secondary to $\mathrm{BPH}$ requiring surgical intervention as determined by their urologist.

- experienced LUTS secondary to BPH $>3$ months in duration.

- had a IPSS value $>12$.

- had a peak urinary flow $<20 \mathrm{~mL} / \mathrm{sec}$ on minimum of $125 \mathrm{~mL}$ voided volume

- had a prostate size, as measured by transrectal ultrasonography (TRUS), $<100 \mathrm{~cm}^{3}$ in volume and prostatespecific antigen (PSA) normal for age group or with a negative TRUS-guided biopsy if PSA is elevated.

- had an American Society of Anesthesiology (ASA) classification of physical status, class 1-3.

- able to read, understand and sign the informed consent.

- is willing and able to comply with all follow-up requirements including multiple follow-up visits.

\section{PVP and TURP protocols}

The PVP procedure was performed using the GreenLight HPS 120 laser system (American Medical Systems). All PVP procedures were performed by investigators familiar with laser surgery of the urinary tract and trained to use the laser system, which was on loan for the study purpose by American Medical Systems. Experience varied from a minimum of 5 cases to over 300 cases. A continuous flow resectoscope and unipolar cautery were used for the TURP procedures. At the end of the procedures, a 3-way catheter with continuous irrigation was inserted. The first procedure was conducted in March 2008 and the last in February 2011.

\section{Effectiveness measures}

In addition to baseline demographic data (age, employment status), several outcomes were collected in the study including the IPSS, ${ }^{10}$ the rate of urine flow (Qmax), PVR volume, measure of serum creatinine, and intra or postoperative complications (e.g., transfusions, changes in hemoglobin, rate of urethral stricture or bladder neck contracture requiring re-operation, re-bleed requiring hospitalization). The Sexual Health Inventory for Men (SHIM) ${ }^{11}$ was used to measure sexual function. All outcomes were measured at baseline and at 1 and 6 month following the procedure. The IPSS was also measured at months 12 and 24 during telephone interviews.

HRQoL was assessed using the EuroQol quality of life questionnaire (EQ-5D), ${ }^{12}$ a preference-based questionnaire which has been widely used. The EQ-5D measures health status in terms of 5 dimensions: (1) mobility, (2) self-care, (3) usual activities, (4) pain/discomfort and (5) anxiety/ depression. Three degrees of impairment are possible: no impairment, some impairment and extreme impairment. The individual responses can be transformed into a utility score on a 0 to 1 scale, in which 0 correspond to death and 1 to a perfect health state. The EQ-5D was administered at baseline and at months 1, 6, 12 and 24 .

\section{Costs and cost-effectiveness analysis}

The assumptions used to calculate the costs associated with the procedures are detailed in Table 1. In addition, resource utilization (e.g., hospitalization, emergency room visits) and productivity loss data (e.g., days missed from work if employed) were prospectively collected. For each patient, the number of resources consumed over the 6-month period was multiplied by the corresponding unit costs and added to the procedure cost. Public sources were used to cost out resource utilization.

As HRQoL is an important outcome of BPH treatment, a cost-utility analysis was conducted to compare PVP and TURP in terms of expected costs and quality-adjusted lifeyears (QALYs) at 6 months. QALYs are a composite measure of outcome where utilities for health states (on 0-1 scale, where 0 corresponds to death and 1 to full health) act as qualitative weights to combine the quantity and quality of 
PVP vs. TURP for LUTS in BPH

\begin{tabular}{|c|c|c|c|}
\hline Item & Estimate & Details & Source \\
\hline Physician costs & $\$ 450.60$ & \#S655 TURP & \\
\hline Anesthesiologist costs & $\$ 161.15$ & $\begin{array}{l}7 \text { basic units, } 4 \text { time units. } \\
\text { Anesthesiologist unit fee }=\$ 14.65\end{array}$ & Services, June 1, 2011. \\
\hline Hospital costs & $\$ 3849.90$ & CCI code: 1QT87BA - TURP & $\begin{array}{l}\text { OCCl, Acute inpatients } 2009 / 2010 \text { by } \\
\text { Procedure }\end{array}$ \\
\hline Device costs: Consumables & $\$ 100.00$ & Cost of resecting loop & Stafinski et al. $2008^{2}$ \\
\hline Total cost per TURP case & $\$ 4561.65$ & & \\
\hline Item & Estimate & Details & Source \\
\hline Physician costs & $\$ 450.60$ & \#S655 TURP & \\
\hline Anesthesiologist costs & $\$ 190.45$ & $\begin{array}{l}7 \text { basic units, } 6 \text { time units. } \\
\text { Anesthesiologist unit fee }=\$ 14.65\end{array}$ & Services, June 1, 2011. \\
\hline Hospital costs & $\$ 1550.94$ & CCl code: 10T59BAAG & OCCI, Day Surgery 2009/2010 \\
\hline Device costs: Capital* & $\$ 242.59$ & & \\
\hline Device costs: Consumables & $\$ 850.00$ & Cost of one fibre & St. Joseph's Healthcare Hamilton \\
\hline Total cost per PVP case & $\$ 3284.58$ & & \\
\hline
\end{tabular}

life. ${ }^{13}$ In the absence of dominance (e.g., PVP more effective and less costly than TURP), the incremental cost per QALY gained was determined. All cost values in the paper were expressed in 2011 Canadian dollars (CAD).

\section{Statistical analyses}

Mean values (standard deviations) and percentages were used to represent continuous and discrete variables, respectively. Statistical significance was assessed with t-tests for continuous variables and chi-square tests for categorical variables. Testing for rare events and non-continuous data produced similar results and are not reported. As the findings of a trial are the results of a single sample drawn from the population, it is important to determine the variability in the costs and outcomes that arises from such sampling. The non-parametric bootstrap, a widely used technique based on sampling with replacement, was used to deal with sampling uncertainty. Uncertainty was also expressed using a cost-effectiveness acceptability curve (CEAC). Using several thresholds (e.g. \$50,000/QALY or \$100,000/QALY), CEACs illustrate the probability that a technology is cost-effective when uncertainty is taken into account.

\section{Results}

\section{Subjects and baseline characteristics}

Between February 2008 and August 2010, 140 PVP subjects and 24 TURP subjects were enrolled in the study. Surgeons with previous PVP experience completed 49 of the PVP pro- cedures and those on the learning curve completed 91 cases. PVP subjects were statistically younger and had a higher Qmax at baseline (Table 2). The two groups were similar with respect to the other variables, including prostate size, prostate volume, flow rate, voiding, PSA and use of medications. Less than $40 \%$ of the population was employed.

\section{Procedure characteristics and complications}

PVP was associated with a longer operating time (63 minutes) than TURP (40.9 minutes) $(p=0.001)$ (Table 3$)$. With only $7.1 \%$ of PVP subjects (i.e., 10 out of 140 subjects) admitted after the procedure, PVP was mainly conducted in an outpatient setting. In contrast all TURP subjects were admitted after the procedure. Although numerically different, the rates of reinsertion of the catheter were not statistically different between the two groups. PVP was associated with statistically less bleeding than TURP as shown by the postoperative hemoglobin and hematocrit values. Post-procedure analgesia was prescribed in 3 TURP subjects $(13 \%)$ and 10 PVP subjects $(7 \%)(p=0.369)$. At 3 months, 1 patient in each treatment arm continued analgesia.

There were no statistical differences between the rate of serious or non-serious adverse events in the 2 groups $(p=0.253)$. Serious adverse events (e.g., hematuria, urinary retention, bleeding) occurred in 6\% of PVP people with no deaths occurring. Although no SAEs were reported in TURP subjects, the difference in SAEs was not statistically significant between the 2 groups $(p=0.201)$. In terms of nonserious adverse events, $12.5 \%$ of TURP subjects and $23.0 \%$ of PVP subjects had a least 1 adverse event $(p=0.253)$. The most frequently reported non-serious adverse events were 
Whelan et al.

\begin{tabular}{lcc}
\hline Table 2. Baseline characteristics & & \\
\hline $\begin{array}{l}\text { Variable name: mean (SD) (minimum, } \\
\text { maximum) }\end{array}$ & TURP, $\mathbf{n = 2 4}$ & PVP, $\mathbf{n = 1 4 0}$ \\
\hline Age (years) & $70.8(7.6)(55,82)$ & $67.4(7.6)(48,85)$ \\
Prostate size (cc) & $54.5(20.5)(19,90)$ & $48.0(18.8)(20,102)$ \\
Prostate volume (cc) & $54.5(22.4)(19,99)$ & $53.8(26.2)(14,152)$ \\
Maximum flow rate (Qmax) (mL/sec) & $8.8(4.4)(2.9,18.4)$ & $11.0(4.2)(2.0,29.8)$ \\
Average flow rate (mL/sec) & $4.7(2.3)(1.7,9.4)$ & $5.6(2.2)(1.4,13.2)$ \\
Total voiding duration (sec) & $58.9(52.5)(9.0,263.0)$ & $51.7(30.4)(15.0,219.0)$ \\
Postvoid residual volume (mL) & $68.8(69.1)(0,233)$ & $106.9(108.5)(0,395)$ \\
PSA (ng/dL) & $3.0(2.1)(0.4,9.1)$ & $2.9(2.5)(0.2,13.4)$ \\
Use of 5-alpha reductase inhibitors (\%) & $11(46 \%)$ & $50(36 \%)$ \\
Use of alpha blockers (\%) & $20(83 \%)$ & $101(72 \%)$ \\
Both alpha and 5-alpha & $8(33 \%)$ & $35(25 \%)$ \\
Employment status (FT or PT) & $5(21 \%)$ & 0.900 \\
\hline
\end{tabular}

TURP: transurethral resection of the prostate; PVP: photoselective vaporization of the prostate; SD: standard deviation; PSA: prostate-specific antigen; FT: full time; PT: part time. Values are reported as mean (SD) (minimum, maximum), or $\mathrm{n}(\%)$.

short-term urinary tract infection $(\mathrm{n}=12)$, urinary retention $(n=10)$, severe dysuria, urgency frequency syndrome $(n=6)$, urethral structure $(n=6)$ and bladder next contracture $(\mathrm{n}=1)$. Re-catheterization was required in 15 individuals (3 TURP [17\%], 12 PVP [(9\%], $p=0.537)$.

\section{Effectiveness measures}

There was no statistically significant difference between the 2 groups in terms of change in the IPSS score at 6 months versus baseline (Table 4). In each group, the IPSS score was reduced by more than $50 \%$ over the 6 -month study period. Improvement in Qmax, PVR and PSA were also observed compared to baseline, although not all differences were statistically or clinically significant (Table 4). Since the use of 5-alpha reductase inhibitors affect PSA, the PSA data is also presented in Table 4 for those individuals who were not on a 5-alpha reductase inhibitors prior to the procedure. Results

\begin{tabular}{|c|c|c|c|}
\hline Variable name & TURP, n=24 & PVP, $n=140$ & $p$ value \\
\hline $\begin{array}{l}\text { Length of procedure } \\
\text { (minutes) }\end{array}$ & $\begin{array}{c}40.9(19.9) \\
(16,95)\end{array}$ & $\begin{array}{c}63.0(24.1) \\
(22,149)\end{array}$ & 0.001 \\
\hline $\begin{array}{l}\text { Admitted after the } \\
\text { procedure? Yes }\end{array}$ & $24(100 \%)$ & $10(7.1 \%)$ & $<0.001$ \\
\hline $\begin{array}{l}\text { Hospitalization days if } \\
\text { admitted }(2 \text { days }= \\
1 \text { overnight stay })\end{array}$ & $\begin{array}{c}2.5(0.5) \\
(2,3)\end{array}$ & $\begin{array}{c}2.0(0.5) \\
(2,3)\end{array}$ & 0.021 \\
\hline $\begin{array}{l}\text { Was the catheter } \\
\text { re-inserted within } 48 \\
\text { hours? Yes }\end{array}$ & $3(13 \%)$ & $6(4 \%)$ & 0.075 \\
\hline $\begin{array}{l}\text { Postoperative hemoglobin } \\
(\mathrm{g} / \mathrm{L})^{*}\end{array}$ & $\begin{array}{c}119.5(16.3) \\
(93,146)\end{array}$ & $\begin{array}{c}140.3(12.1) \\
(108,166)\end{array}$ & $<0.001$ \\
\hline Postoperative hematocrit* & $\begin{array}{l}0.35(0.05) \\
(0.28,0.42)\end{array}$ & $\begin{array}{l}0.41(0.03) \\
(0.32,0.49)\end{array}$ & $<0.001$ \\
\hline $\begin{array}{l}\text { TURP: transurethral resection of the } \\
\text { prostate. *Hemoglobin and hematoc } \\
\text { reported as mean (SD) (minimum, } m\end{array}$ & $\begin{array}{l}\text { te; PVP: photos } \\
\text { re reported in } 60 \\
\text { um), or } n(\%) .\end{array}$ & tive vaporization o & $\begin{array}{l}f \text { the } \\
\text { lues are }\end{array}$ \\
\hline
\end{tabular}

indicate that PSA value decreased significantly more in the TURP arm. The SHIM scores and EQ-5D utility value at 6 months were almost the same as baseline values. Out of a maximum of 0.5 QALYs over 6 months (utility of $1 \times 0.5$ year $=0.5 \mathrm{QALY}$ ), the number of QALYs associated with PVP and TURP were 0.448 (standard deviation [SD]: 0.048) and 0.441 (SD: 0.071), respectively $(p=0.658)$.

\section{Cost-effectiveness analysis}

The two groups were similar, with the exception of physician visits outside of the study protocol and the use of diagnostic tests; these were more frequent in the PVP arm. Of the healthcare resources used, there was only a statistically significant difference in the proportion of patients receiving additional diagnostic tests and procedures (Table 5). The total average costs per patient of the procedure, inpatient admission and follow-up visits (emergency room visits, repeat procedures and visits to family phyicians) up to 6 months was statistically significantly lower for PVP $(p<0.001)$, even though the average follow-up costs for the two groups was greater for PVP. Since only $4 \%$ of patients resumed medications, drug costs were not included in the analysis. Due to the outpatient nature of PVP, treating subjects with PVP instead of TURP significantly decreased the total costs by almost $\$ 1300$ per patient ( $\$ 3284$ vs. $\$ 4562$; $p<0.01$ ) (Table 6). From an economic point of view, PVP is the preferred strategy as it is less costly and generally as effective. As shown in the cost-effectiveness plane, the results of the bootstrap indicated some degree of uncertainty associated with QALYs (Fig. 1). Using two commonly cited cost-effectiveness thresholds for adopting technologies, the probability that PVP was cost-effective was almost greater than 0.99 if decision-makers were willing to pay $\$ 50$ 000/ QALY or $\$ 100$ 000/QALY (Fig. 2).

Since most TURP subjects were not employed at study time, we had limited data to measure productivity losses 
PVP vs. TURP for LUTS in BPH

\begin{tabular}{|c|c|c|c|c|c|}
\hline Clinical measure & Treatment group & Baseline mean (SD) & 6-month mean (SD) & Percentage change & $p$ value \\
\hline \multirow{2}{*}{ IPSS } & PVP & $21.4(6.4)$ & $8.2(6.1)$ & $-62 \%$ & \multirow{2}{*}{0.718} \\
\hline & TURP & $24.4(4.4)$ & $10.5(8.3)$ & $-57 \%$ & \\
\hline \multirow{2}{*}{ Urinary frequency (IPSS 2 hours) } & PVP & $3.7(1.2)$ & $1.7(1.4)$ & $-53 \%$ & \multirow{2}{*}{0.544} \\
\hline & TURP & $3.7(1.2)$ & $2.0(1.5)$ & $-46 \%$ & \\
\hline \multirow{2}{*}{ Max flow rate (Qmax) } & PVP & $11.1(4.2)$ & $17.2(10.1)$ & $+55 \%$ & \multirow{2}{*}{0.705} \\
\hline & TURP & $8.8(4.1)$ & $15.8(8.7)$ & $+79 \%$ & \\
\hline \multirow{2}{*}{ Postvoid residual volume (mL) } & PVP & $106.9(108.5)$ & $30.58(50.2)$ & $-71 \%$ & \multirow{2}{*}{0.018} \\
\hline & TURP & $68.8(69.1)$ & $43.4(69.1)$ & $-31 \%$ & \\
\hline \multirow{2}{*}{ Erectile function (SHIM score) } & PVP & $12.4(7.8)$ & $11.4(8.7)$ & $-7 \%$ & \multirow{2}{*}{0.569} \\
\hline & TURP & $9.4(8.8)$ & $9.3(9.3)$ & $-2 \%$ & \\
\hline \multirow{2}{*}{ PSA } & PVP & $2.9(2.5)$ & $2.8(2.9)$ & $-2 \%$ & \multirow{2}{*}{0.050} \\
\hline & TURP & $3.0(2.1)$ & $2.2(1.7)$ & $-29 \%$ & \\
\hline \multirow{2}{*}{ PSA without pre 5-ARI use } & PVP & $3.2(2.1)$ & $2.6(3.8)$ & $-19 \%$ & \multirow{2}{*}{0.581} \\
\hline & TURP & $2.7(2.0)$ & $1.9(2.0)$ & $-28 \%$ & \\
\hline \multirow{2}{*}{ EQ-5D } & PVP & $0.87(0.1)$ & $0.91(0.1)$ & $+5 \%$ & \multirow{2}{*}{0.134} \\
\hline & TURP & $0.89(0.1)$ & $0.88(0.1)$ & $-1 \%$ & \\
\hline
\end{tabular}

TURP: transurethral resection of the prostate; PVP: photoselective vaporization of the prostate; SD: standard deviation; IPSS: international prostate symptoms score; SHIM: sexual health inventory for men; PSA: prostate-specific antigen; EQ-5D: EuroQol quality of life questionnaire.

associated with TURP $(\mathrm{n}=5)$. Of the 51 PVP subjects, 29 $(57 \%)$ were employed at study time and did not take any days off work following their procedure. For those individuals who did take time off work $(n=22)$, the mean number of days off work was 7.9 (SD: 12.7). In addition, 3 of these 51 subjects $(6 \%)$ reported that their caregiver took an average of 10 days off work.

\section{One-year and two-year outcomes associated with PVP}

At time of writing this paper, 12-month and 24-month follow-up data were available for 134 and 93 PVP subjects, respectively. Out of 134 subjects with 12-month followup, 2 repeat procedures were conducted between 6 and 12 months and $8 \%$ of subjects were back on medications to manage their prostate-related symptoms. The EQ-5D utility increased from 0.907 (SD: 0.119) to 0.926 (SD: 0.117) between 6 and 12 months, while the IPSS improved from 8.20 (SD: 6.13) to 6.56 (SD: 5.39) during the same period. Between 12 and 24 months, 1 repeat procedure was conducted among the 93 subjects with 24-month follow-up, and $11 \%$ were back on prostate-related medications to manage their prostate-related symptoms (mostly anti-cholinergic medications). During the same period, the EQ-5D utility changed from 0.926 (SD: 0.117) to 0.919 (SD: 0.117) and the IPSS score from 6.56 (SD: 5.39) to 6.96 (SD: 6.14).

\section{Discussion}

The search for an ideal procedure to replace the well-established gold standard of TURP has been elusive. This is in part because TURP is an effective, durable procedure with an acceptable morbidity profile. Many alternative procedures have been proposed and tried with variable success. In this study and similar to the findings of others, ${ }^{3-5}$ there was no difference between TURP and PVP in the change in IPSS at 6 months, nor was there a difference in flow rates, HRQoL or sexual function. PVP is a painless, essentially bloodless procedure which can safely be performed in an outpatient setting. With lower 6-month costs and equivalent QALYs, PVP is an attractive technology when compared to TURP. Our 24-month data also showed that the benefits of PVP were durable as both prostate symptoms and HRQoL continued over time.

Our results mirror other trials comparing PVP and TURP. Studies from Lukacs, Capitan and Al-Ansari ${ }^{3,5,14}$ have all shown similar findings with PVP as shown in our study. In a recent editorial, Madersbacher questioned the validity of these trials nicely summarizing the difficulty of recruiting suitable patients in a timely fashion as newer models of the

Table 5. Six-month resource utilization associated with TURP and PVP

\begin{tabular}{|c|c|c|c|}
\hline Cases N (\%) & TURP & PVP & $p$ value \\
\hline Recatherizations & $3(17 \%)$ & $12(9 \%)$ & 0.537 \\
\hline $\begin{array}{l}\text { Repeat or cross-over } \\
\text { procedures }\end{array}$ & $1(4.1 \%)$ & $\begin{array}{c}1 \text { PVP, } 4 \text { TURP } \\
(3.6 \%)\end{array}$ & 0.886 \\
\hline $\begin{array}{l}\text { Emergency department } \\
\text { Visits }\end{array}$ & $1(6 \%)$ & $15(11 \%)$ & 0.318 \\
\hline $\begin{array}{l}\text { Related admissions } \\
\text { (other than procedure) }\end{array}$ & $0(0 \%)$ & $3(2 \%)$ & 0.469 \\
\hline Additional physicians visits & $7(39 \%)$ & $69(49 \%)$ & 0.068 \\
\hline Diagnostic tests/procedures & $2(11 \%)$ & $44(36 \%)$ & 0.020 \\
\hline
\end{tabular}


Whelan et al.

\begin{tabular}{|c|c|c|c|c|}
\hline & TURP costs & PVP costs & Difference & \\
\hline & Mean (SD) (\$) & Mean (SD) (\$) & (TURP-PVP) (\$) & $p$ value \\
\hline Primary procedure & $4562(0)$ & $3284(418)$ & 1167 & $<0.001$ \\
\hline Cross-over treatments $^{\wedge}$ & $0(0)$ & $131(765)$ & -131 & 0.045 \\
\hline Physician costs* & $41(74)$ & 93 (139) & -52 & 0.009 \\
\hline Emergency room visits & $17(85)$ & 87 (293) & -70 & 0.023 \\
\hline Recatheterization & $52(141)$ & 55 (196) & -3 & 0.936 \\
\hline Other hospitalizations & $0(0)$ & 54 (373) & -54 & 0.088 \\
\hline Tests and procedures & $0(1)$ & $53(211)$ & -53 & 0.004 \\
\hline Second procedures & $190(931)$ & 24 (279) & 166 & 0.394 \\
\hline Total costs & $4863(971)$ & $3771(1,315)$ & 971 & 0.001 \\
\hline
\end{tabular}

TURP: transurethral resection of the prostate; PVP: photoselective vaporization of the prostate; SD: standard deviation

*Outside of study visit; ^Impact of 2 TURPs averaged over 140 patients.

technologies evolve. ${ }^{15}$ The authors' response highlights the challenges of trial design in assessing surgical procedures versus drug therapies. ${ }^{15}$ Several limitations should be considered when interpreting the results of our trial. First this was a non-randomized study. As such results should be interpreted with caution although the 2 groups were balanced with respect to clinical characteristics. Randomization was not considered as a part of the study design at the request of the funding agency, as TURP is an insured benefit within Ontario and it was felt that individuals should have access to the reimbursed procedure within the province if they wish. Another study limitation is related to the relatively small number of TURP subjects. In our study, many subjects opted for PVP when offered the choices of PVP or TURP as part of the informed consent process, thus creating several challenges associated with TURP recruitment. The TURP cohort was older and had a slightly lower flow rate at study initiation. The length of stay observed in our TURP cohort was similar to the length of stay of more than 2500 TURPs performed in Ontario in 2008-2009. However, some subgroup analyses were not performed as we did not have enough information on TURP subjects. For example, although our data suggest a quick return to work with PVP, we were not able to compute this number for TURP. Finally, we did not have 24-month data for all PVP and TURP subjects enrolled in the study. The 12- and 24-month data were collected by administering the IPSS in a telephone interview to lower cost and improve numbers of patients, as other authors have reported difficulty in getting patients to return for long-term follow-up.

Patients undergoing PVP showed a decrease in PSA which was less than that shown by other authors, ${ }^{3,5}$ possibly reflecting the fact that over $70 \%$ of the cases were done by surgeons on the learning curve for PVP. One of the authors (JPW) has extensive experience in mentoring community urologists in learning PVP and has found that one of the advantages of PVP is the short learning curve, a combination of online modules, observation and mentoring of 5 cases is sufficient for most urologists.

Recently the manufacturer has introduced a 180-watt PVP laser with a higher maximum power, wider laser beam carrying more energy and more durable fibres. One of the authors (JPW) has completed over 100 cases with this laser and the initial experience is that operative times are shorter and more closely approximate that of TURP.

Despite these limitations, our study provides new information and shows that PVP is a safe, effective and cost-

Fig. 1. Cost-effectiveness plane. 
effective procedure compared to TURP. To the best of our knowledge, this study is the first trial-based cost-effectiveness analysis comparing PVP and TURP.

\section{Conclusion}

In a non-randomized prospective trial of Greenlight PVP versus TURP, there were no differences in the primary outcome of change in IPSS 6 months after surgery. PVP (HPS-120) is an essentially bloodless, painless procedure offering an outpatient alternative to TURP with similar outcomes. As PVP is an outpatient procedure, PVP is also a cost-effective alternative to TURP.

Acknowledgements: The authors would like to thank the Medical Advisory Secretariat and to the Ontario Health Technology Advisory Committee (OHTAC) for their support of the study and for their comments. Drs. O'Reilly, Tarride and Xie are recipients of Career Scientist Awards from the Ontario Ministry of Health and Long-term Care. We would also like to thank Erica Nunes, Craig MacDougald, Meghann Gregg and the research coordinators at each of the sites for their work with the data collection for the study.

Competing interests: Trial Registration: ClinicalTrials.gov: NCT00527371

Ontario Ministry of Health \& Long-term Care (MOHLTC) funding was acquired through an independent Health Technology Assessment and Economic Evaluation Program research grant awarded to Professor Ron Goeree (Grant 06129) through the Programs for Assessment of Technology in Health (PATH Research Institute at St. Joseph's Healthcare Hamilton and to Dr. Jean-Eric Tarride for the specific funding of this study. The results of this field evaluation were presented to OHTAC in November 2011.

This paper has been peer-reviewed.

\section{References}

1. Burke N, Whelan JP, Goeree L, et al. Systematic review and meta-analysis of transurethral resection of the prostate versus minimally invasive procedures for the treatment of benign prostatic obstruction. Urology 2010;75:1015-22. http://dx.doi.org/10.1016/i.urology.2009.08.015

2. Stafinski T, Menon D, Harris $K$, et al. Photoselective vaporization of the prostate for the treatment of benign prostatic hyperplasia. Can Urol Assoc J 2008;2:124-34.

3. Al-Ansari A, Younes N, Sampige VP, et al. GreenLight HPS 120-W laser vaporization versus transurethro resection of the prostate for treatment of benign prostatic hyperplasia: a randomized clinical trial with midterm follow-up. Eur Urol 2010;58:349-55. http://dx.doi.org/10.1016/i.eururo.2010.05.026

4. Bouchier-Hayes DM, Van AS, Bugeja P, et al. A randomized trial of photoselective vaporization of the prostate using the 80-W potassium-itianyl-phosphate laser vs transurethral prostatectomy, with a 1-year follow-up. BJU Int 2010;105:964-9. http://dx.doi.org/10.1111/i.1464-410X.2009.08961.x

5. Capitan C, Blazquez C, Martin MD, et al. GreenLight HPS 120-W laser vaporization versus transurethral resection of the prostate for the treatment of lower urinary tract symptoms due to benign prostatic hyperplasia: a randomized clinical trial with 2-year follow-up. Eur Urol 2011;60:734-9. http://dx.doi. org/10.1016/i.eururo.2011.05.043
6. Goh AC, Gonzalez RR. Photoselective laser vaporization prostatectomy versus transurethral prostate resection: a cost analysis. J Urol 2010;183:1469-73. http://dx.doi.org/10.1016/i.uro.2009.12.020

7. Liatsikos E, Kyriazis I, Kallidonis $P$, et al. Photoselective GreenLight laser vaporization versus transurethral resection of the prostate in Greece: a comparative cost analysis. J Endourol 2012;26:168-73. http:// dx.doi.org/10.1089/end.2011.0089

8. Queen's Printer for Ontario. Ontario Health Technology Advisory Committee http://www.hqontario.ca/ evidence/evidence-process/about-the-ontario-health-technology-advisory-committee. Accessed September 13, 2013.

9. Ontario Ministry of Health and Long-Term Care. Energy delivery systems for treatment of benign prostatic hyperplasia: health technology policy assessment. Toronto: Medical Advisory Secretariat, Ontario Ministry of Health and Long-Term Care; 2006.

10. Barry MJ, Fowler FJ Jr, $0^{\prime}$ Leary MP, et al. The American Urological Association symptom index for benign prostatic hyperplasia. The Measurement Committee of the American Urological Association. J Urol 1992;148:1549-57.

11. Rosen RC, Cappelleri JC, Smith MD, et al. Development and evaluation of an abridged, 5-ttem version of the International Index of Erectile Function (IIIEF-5) as a diagnostic tool for erectile dysfunction. Int J Impot Res 1999;11:319-26. http://dx.doi.org/10.1038/si.iji.3900472

12. Dolan P. Modeling valuations for EuroQol health states. Med Care 1997;35:1095-108. http://dx.doi. org/10.1097/00005650-199711000-00002

13. Weinstein MC, Stason WB. Foundations of cost-effectiveness analysis for health and medical practices. $N$ Engl J Med 1977;296:716-21. http://dx.doi.org/10.1056/NEJM197703312961304

14. Lukacs B, Loeffler J, Bruyere F, et al. Photoselective vaporization of the prostate with Greenlight 120-W kaser compared with monopolar transurethral resection of the prostate:a multicenter randomized controlled trial. Eur Urol 2012;61:1165-73. http://dx.doi.org/10.1016/i.eururo.2012.01.052

15. Madersbacher S, After Three Randomized Trials Comparing 120-W HPS KTP laser vaporization to Transurethral Resection of the Prostate, Is this procedure finally first line, outdated or still not surpassing TURP? Eur Urol 2012;61:1174-6; discussion 1176-7. http://dx.doi.org/10.1016/i.eururo.2012.02.046. Epub 2012 Feb 25.

Correspondence: Dr. J. Paul Whelan, PATH Research Institute, 25 Main St. West, Suite 2000, Hamilton, 0N L8P 1HI; fax: 905-522-0568; whelanp@mcmaster.ca 